\title{
RECURRÊNCIA DA HEPATITE C APÓS TRANSPLANTE HEPÁTICO DE DOADOR VIVO E FALECIDO
}

\author{
Júlio Cezar Uili COELHO'1, Luciano OKAWA'1, Mônica Beatriz PAROLIN'1, \\ Alexandre Coutinho Teixeira de FREITAS ${ }^{1}$, Jorge E. Fouto MATIAS ${ }^{1}$ e Alysson Rogério MATIOSKI²
}

\begin{abstract}
RESUMO - Objetivo - Determinar a recurrência da hepatite $\mathrm{C}$ em pacientes submetidos a transplante hepático de doador vivo comparados com os submetidos a transplante hepático de doador falecido. Métodos - Do total de 333 transplantes hepáticos, 279 (83,8\%) eram de doador falecido e 54 (16,2\%) de doador vivo. Hepatopatia crônica pelo vírus da hepatite C foi a indicação mais comum tanto de transplante hepático de doador falecido ( 82 pacientes) como de doador vivo (19 pacientes). O protocolo de estudo eletrônico de todos pacientes com hepatopatia crônica pelo vírus da hepatite $\mathrm{C}$ foi avaliado. Os dados coletados foram analisados estatisticamente conforme a idade, sexo, resultado dos exames laboratoriais, recidiva do vírus da hepatite $\mathrm{C}$ e rejeição aguda. Resultados - O total de 55 transplantes hepáticos de doador falecido e 10 de doador vivo realizados em pacientes com cirrose hepática pelo vírus da hepatite $\mathrm{C}$, foi incluído no estudo. As características clínicas e laboratoriais pré-transplante dos dois grupos foram similares, exceto o tempo de atividade de protrombina que foi maior no grupo de transplante hepático de doador falecido do que no de doador vivo $(P=0,04)$. A recidiva da hepatite $\mathrm{C}$ foi similar nos grupos de transplante hepático de doador falecido $(\mathrm{n}=37 ; 69,3 \%)$ e de doador vivo $(\mathrm{n}=7 ; 70 \%)(P=0,8)$. A incidência de rejeição aguda foi igual no grupo de transplante hepático de doador falecido $(\mathrm{n}=27 ; 49 \%)$ e no grupo de doador vivo $(\mathrm{n}=2 ; 20 \%)(P=0,08)$. A recurrência do vírus da hepatite $\mathrm{C}$ nos pacientes do grupo de transplante hepático de doador falecido que receberam pulsoterapia ( 9 de 11 pacientes) foi similar aos demais pacientes (28 de 44 pacientes) $(P=0,25)$. A recurrência também foi similar nos pacientes do grupo de transplante hepático de doador vivo que receberam pulsoterapia ( 1 de 1 paciente) em relação aos que não receberam (6 de 9 pacientes) $(P=0,7)$. Conclusães - A recurrência do vírus da hepatite $\mathrm{C}$ é similar nos receptores de transplante hepático de doador falecido e de doador vivo.
\end{abstract}

DESCRITORES - Hepatite C. Transplante de fígado. Doadores vivos. Doadores de tecidos.

\section{INTRODUÇÃO}

A hepatopatia crônica causada pelo vírus da hepatite C (VHC) é a indicação mais comum de transplante hepático tanto intervivos como cadavérico em vários países, inclusive no Brasil ${ }^{(7,16,28)}$. A recurrência da hepatite C após transplante hepático (TH) é elevada, podendo evoluir para cirrose e perda do enxerto em elevada proporção dos pacientes ${ }^{(5,9,15,24)}$. Estas complicações fazem com que o resultado do TH no paciente com hepatite $\mathrm{C}$, tanto em sobrevida do enxerto como do paciente, seja inferior a de pacientes transplantados sem hepatite $\mathrm{C}^{(1,3,4,20,23)}$.

Uma das estratégias adotadas em virtude da crescente disparidade entre o número de pacientes em lista de espera e o número de doações de órgãos, é o transplante hepático de doador vivo (THDV) ${ }^{(6,14)}$. Esta modalidade, além de reduzir o tempo de espera e a mortalidade na lista de espera emprega enxerto potencialmente de melhor qualidade (doador saudável e menor tempo de isquemia fria) ${ }^{(7,14,26)}$. Entretanto, alguns estudos sugerem que a recurrência do VHC é mais precoce ou mais agressiva após o THDV do que após o transplante hepático de doador falecido (THDF) $)^{(8,9,21,27)}$.

O objetivo deste estudo foi determinar a recurrência da hepatite $\mathrm{C}$ em pacientes submetidos a THDV comparados com os submetidos a THDF.

\section{MÉTODOS}

O total de 333 transplantes hepáticos foi realizado no Hospital de Clínicas da Universidade Federal do Paraná, Curitiba, PR, no período de setembro de 1991 a dezembro de 2005. Desses, 279 (83,8\%) eram THDF e $54(16,2 \%)$ THDV. Hepatopatia crônica pelo VHC foi a indicação mais comum de THDF (82 pacientes) bem como de THDV (19 pacientes). O protocolo de estudo eletrônico de todos pacientes com hepatopatia crônica pelo VHC foi avaliado. Foram excluídos os pacientes com os seguintes critérios: 1) seguimento clínico e/ou laboratorial inferior a 3 meses (perda de seguimento ou óbito); 2) não disponibilidade de teste de PCR qualitativo

Trabalho realizado no Serviço de Cirurgia do Aparelho Digestivo e Transplante Hepático do Hospital de Clínicas da Universidade Federal do Paraná

${ }^{1}$ Serviço de Cirurgia do Aparelho Digestivo e Transplante Hepático do Hospital de Clínicas da Universidade Federal do Paraná; ${ }^{2}$ Setor de Ciências da Saúde da Universidade Federal do Paraná, Curitiba, PR

Correspondência: Prof. Júlio Coelho - Rua Bento Viana, 1140 - apt. 2202 - 80240-110 - Curitiba, PR. E-mail: coelhojcu@yahoo.com.br 
para hepatite $\mathrm{C}$ realizado pós-TH; 3) não disponibilidade de pelo menos, uma biopsia hepática após o $\mathrm{TH}$; 4) presença de coinfecção pelo vírus da hepatite B e/ou HIV antes e após o $\mathrm{TH}$; 5) diagnóstico de rejeição celular aguda persistente ou rejeição crônica; 6) transplante duplo de rim e fígado; e 7) dados incompletos no protocolo de estudo.

\section{Diagnóstico de recurrência de infecção pelo VHC}

O diagnóstico da recurrência do VHC no enxerto hepático foi suspeitado clinicamente (alteração das enzimas hepáticas, ultrassonografia com Doppler de vasos hepáticos normal, estudos das vias biliares normal por colangiorressonância magnética, imunossupressão adequada e exclusão de exposição a drogas hepatóxicas) e confirmado pela presença de RNA-VHC detectado por teste de PCR qualitativo e pelo exame histológico de tecido hepático obtido por biopsia percutânea. As alterações histológicas indicativas de recurrência viral consistiram da presença de atividade lobular (corpos eosinofílicos, infiltrado linfocitário no lóbulo e lesão hepatocelular) e inflamação portal, sem a presença de características diagnósticas de rejeição ${ }^{(19,29)}$. Todas as biopsias foram interpretadas por um patologista com experiência em histopatologia de $\mathrm{TH}$.

\section{Imunossupressão}

Todos os pacientes submetidos a transplante hepático receberam esquema de imunossupressão composto por um inibidor da calcineurina (ciclosporina ou tacrolimo) associado a corticosteróide e uma droga antiproliferativa (azatioprina $1 \mathrm{mg} / \mathrm{kg} /$ dia ou micofenolato mofetil $1 \mathrm{~g}$ de 12/12 horas, por via oral). O esquema de imunossupressão variou conforme o período de tempo em que os pacientes foram transplantados. $\mathrm{O}$ inibidor de calcineurina utilizado até 1998 foi a ciclosporina em microemulsão (4 mg/kg de 12/12 horas por via oral, objetivando nível sérico entre 200 e $350 \mathrm{ng} / \mathrm{mL}$ no primeiro ano e, posteriormente, 120 a $200 \mathrm{ng} / \mathrm{mL}$ ). Após 1999, a ciclosporina foi substituída pelo tacrolimo $(0,05 \mathrm{mg} / \mathrm{kg}$ de $12 / 12$ horas por via oral, objetivando nível sérico de 10 a $15 \mathrm{ng} / \mathrm{mL}$ no primeiro mês, 8 a $12 \mathrm{ng} / \mathrm{mL}$ até o $4^{\circ}$ mês e após, 6 a $10 \mathrm{ng} / \mathrm{mL}$ ).

O protocolo de administração de corticosteróide incluía a administração de $1 \mathrm{~g}$ de metilprednisolona por via endovenosa após a reperfusão do enxerto, iniciando $50 \mathrm{mg}$ por via endovenosa no $1^{\circ}$ dia pós-transplante e reduzindo gradualmente até $10 \mathrm{mg} / \mathrm{dia}$ de prednisolona no $30^{\circ}$ dia e $5 \mathrm{mg} /$ dia após o $3^{\circ}$ mês. Até 1998 o corticosteróide era mantido em doses baixas ( $5 \mathrm{mg} / \mathrm{dia})$, por no mínimo 1 ano após o transplante, sendo que a partir de 1999 a prednisolona foi suspensa após o $4^{\circ}$ mês. O micofenolato mofetil substituiu a azatioprina após o ano 2000, sendo empregado durante os 2 primeiros meses após o transplante, ou por períodos mais longos quando não era possível manter níveis terapêuticos dos inibidores da calcineurina.

Os episódios de rejeição aguda do enxerto foram diagnosticados e graduados de acordo com a classificação de $\operatorname{Banff}^{(2)}$. As formas leves foram tratadas com aumento da dose dos inibidores de calcineurina, associado ou não à introdução do micofenolato mofetil. As formas moderadas ou severas foram tratadas com pulsoterapia de corticosteróides (três doses de $1 \mathrm{~g}$ de metilprednisolona administradas por via endovenosa em dias alternados).

\section{Dados avaliados e análise estatística}

O trabalho foi aprovado pelo Comitê de Ética em Pesquisa do Hospital de Clínicas da Universidade Federal do Paraná. Os dados coletados foram analisados conforme a idade, sexo, resultado dos exames laboratoriais, recidiva do VHC e rejeição aguda. As variáveis contínuas foram expressas com média \pm desvio padrão e as variáveis categóricas como percentagens. A análise estatística foi realizada com o programa Excel, empregando-se o teste $t$ de Student para as variáveis contínuas, o teste do qui ao quadrado para as variáveis binomiais com n maior ou igual a 20 e o teste de Fisher para as variáveis binomiais com n menor do que 20. Foi considerado estatisticamente significativo valores de $P \leq 0,05$.

\section{RESULTADOS}

O total de 55 THDF e 10 THDV realizados em pacientes com cirrose hepática pelo VHC foi incluído no estudo. Foram excluídos do estudo 36 pacientes (27 THDF e 9 do THDV), sendo 26 devido a óbito nos primeiros 3 meses após transplante (18 THDF e 8 THDV); 4 por co-infecção pelo vírus da hepatite $\mathrm{B}$ (3 THDF e 1 THDV); 4 THDF por seguimento clínico e laboratorial inferior a 3 meses; e 2 THDF devido a dados incompletos no protocolo de estudo.

As características clínicas e laboratoriais dos dois grupos antes do TH estão descritas na Tabela 1. A idade média dos pacientes do grupo de THDF foi de 51,9 \pm 8,7 anos e do grupo de THDV $50,1 \pm 7,0$ anos. Não houve diferença entre os dois grupos quanto à idade, sexo, níveis séricos de creatinina e bilirrubina. O tempo de atividade de protrombina (TAP) foi maior no grupo de THDF do que no THDV $(P=0,04)$.

TABELA 1. Características clínicas e laboratoriais dos pacientes submetidos a THDF e THDV antes do transplante

\begin{tabular}{lccc}
\hline & THDF & THDV & $\boldsymbol{P}$ \\
\hline Número de pacientes & 55 & 10 & \\
Idade média (anos) & $51,9 \pm 8,7$ & $50,1 \pm 7,0$ & 0,8 \\
Sexo masculino & 35 & 6 & \\
Albumina sérica $(\mathrm{g} / \mathrm{dL})$ & $2,62 \pm 0,24$ & $2,63 \pm 0,29$ & 0,9 \\
Creatinina sérica $(\mathrm{mg} / \mathrm{dL})$ & $1,27 \pm 0,22$ & $1,1 \pm 0,23$ & 0,07 \\
TAP $($ seg) & $16,4 \pm 2,17$ & $14,9 \pm 1,8$ & 0,04 \\
\hline
\end{tabular}

THDF= transplante hepático de doador falecido

$T H D V=$ transplante hepático de doador vivo

TAP = tempo de atividade de protrombina

A recurrência do VHC foi similar nos grupos de THDF e THDV $(P=0,8)$. O total de 37 pacientes $(69,3 \%)$ do grupo de THDF apresentou recidiva do VHC, sendo que o diagnóstico foi estabelecido em média em 20,1 $\pm 21,7$ meses após o TH. Dos pacientes do grupo de THDV, sete $(70 \%)$ tiveram recidiva em média 11,3 \pm 9,5 meses após o TH. O período de seguimento médio dos pacientes do grupo de THDF foi de 53,4 \pm 40,5 meses e do grupo de THDV foi de 37,4 $\pm 16,6$ meses. 
A incidência de rejeição aguda foi igual no grupo de THDF $(\mathrm{n}=27 ; 49 \%)$ e no grupo de THDV $(\mathrm{n}=2 ; 20 \%)(P=0,08)$. A maioria das rejeições agudas do grupo de THDF $(n=16 ; 59,3 \%)$, e em apenas um paciente do grupo de THDV (50\%), foi leve e tratada com elevação da dose de ciclosporina ou tacrolimo, acompanhada ou não da adição de micofenolato. Os demais episódios de rejeição aguda dos dois grupos (11 THDF e 1 THDV) foram eficazmente tratados com três doses de pulsoterapia de metilprednisolona. A recidiva do VHC nos pacientes do grupo de THDF que receberam pulsoterapia ( 9 de 11 pacientes) foi similar a dos demais pacientes ( 28 de 44 pacientes) $(P=0,25)$. A recidiva também foi similar nos pacientes do grupo de THDV que receberam pulsoterapia (um de um paciente) em relação aos que não receberam (seis de nove pacientes) $(P=0,7)$.

\section{DISCUSSÃO}

Recurrência do VHC após TH é um dos tópicos mais importantes da Hepatologia por vários motivos. Primeiro, a cirrose hepática causada pelo VHC é a indicação mais comum de TH nos Estados Unidos, Europa e Brasil ${ }^{(4,11)}$. Segundo, a recurrência do VHC ocorre na quase totalidade dos receptores ${ }^{(17,25)}$. Terceiro, a progressão da doença hepática causada pelo VHC no fígado transplantado é mais rápida e agressiva do que a observada no fígado nativo ${ }^{(4,20)}$. Cirrose hepática avançada ocorre em poucos anos em número expressivo de pacientes transplantados por hepatopatia pelo $\mathrm{VHC}^{(10,20)}$. Finalmente, retransplante por cirrose hepática causada pela recurrência do VHC é associada a prognóstico ruim $^{(4,20)}$. Assim, alguns serviços contraindicam retransplante hepático nos casos de recurrência do $\mathrm{VHC}^{(11)}$.

Como não existe nenhum esquema profilático eficaz, é importante determinar os fatores de risco de recurrência do VHC após TH para que se possa, mediante intervenções apropriadas, reduzir a taxa e a agressividade dessa complicação. Um dos fatores de risco mais importantes é o esquema de imunossupressão, principalmente com o uso de corticóides em doses elevadas, $\mathrm{OKT}_{3}$ (anticorpo monoclonal) e globulina antilinfocítica para tratar rejeição hepática celular (rejeição aguda) ${ }^{(10,22)}$. Recentemente, alguns autores sugeriram que o THDV é associado a maior risco de recurrência do VHC do que o $\operatorname{THDF}^{(8,9,21,27)}$. SCHIANO et al. ${ }^{(21)}$ relataram maior replicação do $\mathrm{VHC}$ em receptores de THDV, cujos possíveis mecanismos são: 1) proliferação celular acelerada nos receptores do THDV causaria alterações celulares que facilitariam a entrada do VHC nos hepatócitos ou promoveriam a replicação viral; 2) células hepáticas com estado funcional superior; 3 ) inoculação viral maior devida à captação mais eficiente e a menor perda durante o transplante; e 4) o menor tamanho do enxerto reduziria a capacidade de metabolizar medicamentos, resultando em maior imunossupressão e menor defesa antiviral. Outros mecanismos foram sugeridos, incluindo a hipótese de que a maior compatibilidade HLA entre doador e receptor nos THDV facilitaria a recurrência do VHC neste tipo de transplante ${ }^{(25)}$.

O presente estudo mostrou que a taxa de recurrência do VHC é igual após THDF e THDV. HUMAR et al. ${ }^{(11)}$ também relataram que a taxa de recurrência do VHC pós-THDF é semelhante à observada pós-THDV e pós-"split liver" (fígado bipartido), ou seja, de doadores que foram submetidos a ressecção hepática. Estes dados sugerem que a regeneração hepática não altera a recurrência de hepatite $\mathrm{C}$ como inicialmente foi hipotetizado. Outros autores também não observaram diferença na recurrência do VHC entre THDF e THDV $(10,17,25,28)$. Outro estudo recente demonstrou que a sobrevida do paciente e do enxerto é similar após THDF e THDV ${ }^{(18)}$. Os achados desse estudo são particularmente importantes porque são baseados nos dados do Registro Científico de Receptores de Transplantes dos Estados Unidos desde 1999, o maior número de transplantes hepáticos avaliados até o presente. A discrepância dos estudos anteriores com os atuais pode ser devida ao pequeno número de THDV incluídos nos estudos iniciais.

A disfunção hepática é geralmente menos intensa nos receptores de THDV do que nos de $\operatorname{THDF}^{(13,18)}$. As características clínicas e laboratoriais dos pacientes dos dois grupos foram similares no presente estudo, exceto pelo TAP que era mais elevado no grupo de THDV. Dados similares foram obtidos em outros estudos $^{(13,18)}$.

O esquema de imunossupressão pode influenciar a replicação do $\operatorname{VHC}^{(9,12)}$. GARCIA-RETORTILLO et al..$^{(9)}$ relataram que 5 de 7 pacientes que não receberam corticóide no esquema de imunossupressor pós-TH tiveram redução na carga viral do VHC, enquanto redução similar foi observada em somente 1 de 13 pacientes que receberam corticóide. Estes dados sugerem que a ausência de corticóide no esquema imunossupressor pós-TH pode reduzir a replicação viral. No presente estudo, o mesmo esquema imunossupressor foi utilizado nos receptores de THDF e de THDV. A percentagem de pacientes que apresentou rejeição celular (rejeição aguda) foi similar nos dois grupos desta série. Estes dados minimizam a influência dos imunossupressores nos resultados deste estudo.

\section{CONCLUSÃO}

A recidiva do VHC é similar nos receptores de THDF e THDV. Os dados desse e de outros estudos recentes sugerem que a presença do VHC não deve influenciar na decisão de indicação de THDV. 
Coelho JCU, Okawa L, Parolin MB, Freitas ACT, Matias JEF, Matioski AR. Hepatitis C recurrence after living donor and cadaveric liver transplantation. Arq Gastroenterol. 2009;46(1): 38-42.

ABSTRACT - Objective - To determine the recurrence of hepatitis C in patients subjected to living donor liver transplantation compared to those subjected to cadaveric liver transplantation. Methods - Of a total of 333 liver transplantations, 279 (83.8\%) were cadaveric liver transplantation and 54 (16.2\%) living donor liver transplantation. Hepatic cirrhosis due to hepatitis $\mathrm{C}$ virus was the most common indication of both cadaveric liver transplantation ( 82 patients) and living donor liver transplantation (19 patients). The electronic study protocols of all patients with hepatic cirrhosis due to hepatitis $\mathrm{C}$ virus were reviewed. All data, including patients' age and sex, laboratory tests, hepatitis C virus recurrence and acute rejection were evaluated statistically. Results - A total of 55 cadaveric liver transplantation and 10 living donor liver transplantation performed in patients with liver cirrhosis due to hepatitis $\mathrm{C}$ virus was included in the study. Clinical and laboratory characteristics of the two groups before the transplantation were similar, except for the prothrombin time that was higher for the cadaveric liver transplantation than the living donor liver transplantation $(P=0.04)$. Hepatitis $\mathrm{C}$ virus recurrence was similar in the cadaveric liver transplantation $(\mathrm{n}=37 ; 69.3 \%)$ and living donor liver transplantation $(\mathrm{n}=7 ; 70 \%)$ groups $(P=0.8)$. The incidence of acute rejection was similar in cadaveric liver transplantation $(\mathrm{n}=27 ; 49 \%)$ and living donor liver transplantation $(\mathrm{n}=2 ; 20 \%)$ groups $(P=0.08)$. Hepatitis $\mathrm{C}$ virus recurrence in patients of the cadaveric liver transplantation group who received bolus doses of corticosteroids ( 9 of 11 patients) was similar to the remained patients ( 28 of 44 patients) $(P=0.25)$. Recurrence was also similar in patients of the living donor liver transplantation group who received bolus doses of corticosteroids (one of one patient) in relation to those who did not receive them (six of nine patients) $(P=0.7)$. Conclusion - Hepatitis $\mathrm{C}$ recurrence is similar in patients who underwent living donor liver transplantation or cadaveric liver transplantation.

HEADINGS - Hepatitis C. Liver transplantation. Living donors. Tissue donors.

\section{REFERÊNCIAS}

1. Bahra M, Neumann UP, Jacob D, Berg T, Neuhaus R, Langrehr JM, Neuhaus P. Outcome after liver re-transplantation in patients with recurrent chronic hepatitis $\mathrm{C}$. Transpl Int. 2007;20:771-8.

2. Banff schema for grading liver allograft rejection: an international consensus document. Hepatology, 1997;25:658-63.

3. Berenguer M. Management of hepatitis $\mathrm{C}$ virus in the transplant patient. Clin Liver Dis. 2007;11:355-76.

4. Berenguer M. Recurrent hepatitis C: worse outcomes established, interventions still inadequate. Liver Transpl. 2007;13:641-3.

5. Bittencourt PL, Silva RCSMA, Pessoa MG, Marroni CA. Resultado do inquérito nacional sobre condutas no acompanhamento e no tratamento da recurrência da hepatite $\mathrm{C}$ em portadores de vírus $\mathrm{C}$ submetidos a transplante de fígado. Arq Gastroenterol. 2007; 44:78-84.

6. Coelho JCU, Parolin MB, Baretta GAP, Pimentel SK, Freitas ACT, Colman D. Qualidade de vida do doador após transplante hepático intervivos. Arq Gastroenterol. 2005; $42: 83-8$.

7. Coelho JCU, de Freitas ACT, Matias JEF, Zeni-Neto C, de Godoy JL, Parolin MB, Okawa L. Donor complications including the report of one death in right-lobe living donor liver transplantation. Dig Surg. 2007;24:191-6.

8. Gaglio PJ, Malireddy S, Levitt BS, Lapointe-Rudow D, Lefkowitch J, Kinkhabwala M, Russo MW, Emond JC, Brown Jr RS. Increased risk of cholestatic hepatitis $\mathrm{C}$ in recipients of grafts from living versus cadaveric liver donors. Liver Transpl. 2003;9:1028-35.

9. Garcia-Retortillo M, Forns X, Llovet JM, Navasa M, Feliu A, Massaguer A, Bruguera M, Fuster J, Garcia-Valdecasas JC, Rimola A. Hepatitis C recurrence is more severe after living donor compared to cadaveric liver transplantation. Hepatology. 2004;40:699-707.

10. Guo L, Orrego M, Rodriguez-Luna H, Balan V, Byrne T, Chopra K, Douglas DD, Harrison E, Moss A, Reddy KS, Williams JW, Rakela J, Mulligan D, Vargas HE. Living donor liver transplantation for hepatitis C-related cirrhosis: no difference in histological recurrence when compared to deceased donor liver transplantation recipients. Liver Transpl. 2006;12:560-5.

11. Humar A, Horn K, Kalis A, Glessing B, Payne WD, Lake J. Living donor and splitliver transplants in hepatitis $\mathrm{C}$ recipients: does liver regeneration increase the risk for recurrence. Am J Transpl 2005;5:399-405.

12. Ichikawa T, Nakao K, Hamasaki K, Honda T, Shibata H, Akahoshi M, Eguchi S, Takatsuki M, Kanematsu T, Eguchi K. Clearance of hepatitis C virus after living-donor liver transplantation in spite of residual viremia on end date of interferon therapy before transplantation. World J Gastroenterol. 2007;13:4149-51.

13. Kam I. Adult-adult right hepatic lobe living donor liver transplantation for status $2 \mathrm{a}$ patients: too little, too late. Liver Transpl. 2002;8:347-9.
14. Neto JS, Carone E, Pugliese V, Salzedas A, Fonseca EA, Teng H, Porta G, Pugliese R, Miura I, Baggio V, Hayashi M, Beloto M, Guimaraes T, Godoy A, Kondo M, Chapchap P. Living donor liver transplantation for children in Brazil weighing less than 10 kilograms. Liver Transpl. 2007;13:1153-8.

15. Ono-Nita SK, Nita ME, Carrilho FJ. Hepatite C. Fatos em números. Arq Gastroenterol. 2006;43:71-2.

16. Patel S, Orloff M, Tsoulfas G, Kashyap R, Jain A, Bozorgzadeh A, Abt P. Living-donor liver transplantation in the United States: identifying donors at risk for perioperative complications. Am J Transplant. 2007;7:2344-9.

17. Rodrigues-Luna H, Vargas HE, Sharma P, Ortiz J, de Petris G, Balan V, Byrne T, Moss A, Mulligan D, Rakela J, Douglas DD. Hepatitis C virus recurrence in living donor liver transplant recipients. Dig Dis Sci. 2004;49:38-41.

18. Russo MW, Galanko J, Beavers K, Fried MW, Shrestha R. Patient and graft survival in hepatitis $\mathrm{C}$ recipients after adult living donor liver transplantation in the United States. Liver Transpl. 2004;10:340-6.

19. Samuel D, Forns X, Berenguer M, Trautwein C, Burroughs A, Rizzetto M, Trepo C. Report of the Monothematic EASL Conference on Liver Transplantation (Paris, France, January 12-14, 2006). J Hepatol. 2006;45:127-43.

20. Samuel D, Roche B. Ribavirin in the treatment of recurrent hepatitis $\mathrm{C}$ after liver transplantation: difficult to manage but essential for success. J Hepatol. 2007;46:988-91.

21. Schiano TD, Gutierrez JA, Walewski JL, Fiel MI, Cheng B, Bodenheimer Jr H, Thung SN, Chung RT, Schwartz ME, Bodian C, Branch AD. Accelerated hepatitis C virus kinetics but similar survival rates in recipients of liver graft from living versus deceased donors. Hepatology. 2005;42:1420-8.

22. Shifman ML, Stravitz RT, Contos MJ, Mills AS, Sterling RK, Luketic VA, Sanyal AJ, Cotterell D, Maluf D, Posner MP, Fisher RA. Histologic recurrence of chronic hepatitis $\mathrm{C}$ virus in patients after living donor and deceased donor liver transplantation. Liver Transpl. 2004;10:1248-55.

23. Shiffman ML. Treating chronic hepatitis $C$ virus after liver transplantation: balancing the risks against the chance for success. Liver Transpl. 2007;13:1088-91.

24. Stanca CM, Fiel MI, Kontorinis N, Agarwal K, Emre S, Schiano TD. Chronic ductopenic rejection in patients with recurrent hepatitis $\mathrm{C}$ virus treated with pegylated interferon alfa-2a and ribavirin. Transplantation. 2007;84:180-6.

25. Takada Y, Haga H, Ito T, Nabeshima M, Ogawa K, Kasahara M, Oike F, Ueda M, Egawa $\mathrm{H}$, Tanaka K. Clinical outcomes of living donor liver transplantation for hepatitis C virus (HCV)-positive patients. Transplantation. 2006;81:350-4.

26. Trotter JF, Wisniewski KA, Terrault NA, Everhart JE, Kinkhabwala M, Weinrieb RM, Fair JH, Fisher RA, Koffron AJ, Saab S, Merion RM, A2ALL Study Group. Outcomes of donor evaluation in adult-to-adult living donor liver transplantation. Hepatology. 2007;46:1476-84.

27. Trotter J, Schiano T, Kim-Schluger L. Living donor liver transplantation in hepatitis C patients short-term results compared to cadaveric transplantation. Am J Transpl. 2001;1(suppl):316A. 
28. Van Vlierberche, Troisi R, Colle I, Ricciardi S, Praet M, de Hemptinne B. Hepatitis $\mathrm{C}$ infection-related liver disease: patterns of recurrence and outcome in cadaveric and living-donor liver transplantation in adults. Transplantation. 2004;77:210-4.

29. Wiesner RH, Sorrell M, Villamil F, International Liver Transplantation Society Expert Panel. Report of the first international Liver Transplantation Society expert panel consensus conference on liver transplantation and hepatitis C. Liver Transpl. 2003;9(suppl 3):S1-9.

Recebido em 22/4/2008 Aprovado em 8/7/2008. 